\title{
Michel BIARD (dir.), Les politiques de la Terreur
}

\section{3-1794}

Rennes, Presses Universitaires de Rennes et Paris, SER, 2008, 484 p., ISBN

978-7535-0601-5, $23 €$

\section{Guillaume Mazeau}

\section{(2) OpenEdition}

Journals

Édition électronique

URL : https://journals.openedition.org/ahrf/11583

DOI : $10.4000 /$ ahrf.11583

ISSN : 1952-403X

Éditeur :

Armand Colin, Société des études robespierristes

Édition imprimée

Date de publication : 1 octobre 2009

Pagination : 197-200

ISBN : 978-2-200-92560-4

ISSN : 0003-4436

Référence électronique

Guillaume Mazeau, « Michel BIARD (dir.), Les politiques de la Terreur 1793-1794 », Annales historiques de la Révolution française [En ligne], 358 | octobre-décembre 2009, mis en ligne le 29 juillet 2010, consulté le 23 avril 2022. URL : http://journals.openedition.org/ahrf/11583 ; DOI : https://doi.org/10.4000/ahrf. 11583

Ce document a été généré automatiquement le 23 avril 2022.

Tous droits réservés 


\section{Michel BIARD (dir.), Les politiques de la Terreur 1793-1794}

Rennes, Presses Universitaires de Rennes et Paris, SER, 2008, 484 p., ISBN

978-7535-0601-5, $23 €$

Guillaume Mazeau

\section{RÉFÉRENCE}

Michel BIARD (dir.), Les politiques de la Terreur 1793-1794, Rennes, Presses Universitaires de Rennes et Paris, SER, 2008, 484 p., ISBN 978-7535-0601-5, $23 €$

1 La volée de bois vert infligée par la critique au Livre Noir de la Révolution française, paru le 21 janvier 2008 ( !), est un signe : les anathèmes ressassés depuis deux cents ans sur la Terreur sont usés jusqu'à la corde. Le colloque organisé en 2007 à Rouen par le GRHis (Université de Rouen) et la Société des études robespierristes, montre que l'histoire s'est refroidie. Il est possible de revenir, en France, à tête reposée et treize ans après le volume dirigé par Keith Michael Baker - The French Revolution and the Creation of Modern Political Culture. vol. 4, The Terror, Oxford, Pergamon Press, 1994 -, sur un des principaux mythes fondateurs de la République.

2 L'entreprise ne cache pas son ambition : couvrir, à travers une trentaine d'articles, le plus d'aspects possibles des rouages de la Terreur. Le grand angle vaut argument épistémologique: alors que l'on continue parfois de réduire la Terreur à la seule question de la violence d'État, Michel Biard affirme que pour bien la comprendre il faut au contraire regarder ses effets les plus contradictoires, à différentes échelles. Autrement dit, au lieu du réductionnisme monologique suggéré par l'expression « la » politique de la Terreur, il faudrait plutôt parler « des » politiques de la Terreur, cellesci étant définies comme tous les moyens mis en œuvre pour défendre la Révolution (Patrice Gueniffey, La politique de la Terreur. Essai sur la violence révolutionnaire, 1789-1794, Paris, Fayard, 2000). Dans cette acception, la répression politique fait partie d'un 
ensemble de mesures qui comprend la promotion de la démocratie ou les politiques économiques, sociales (assistance, éducation) et culturelles.

Dans la première partie consacrée aux rouages de la Terreur, les contributeurs mettent en valeur le rôle des institutions et acteurs intermédiaires, nuancent le caractère extralégal des lois révolutionnaires et s'écartent des analyses systémiques. L'introduction de Michel Biard et Christine Peyrard invite à ouvrir la chronologie, insistant sur les décisions du printemps 1793, puis du 10 octobre 1793 et du 14 frimaire an II, ce qui relativise l'impact des journées des 4 et 5 septembre 1793, pourtant traditionnellement mises en avant. La Terreur ne serait pas cette parenthèse honnie, cette période fermée sur elle-même que l'on décrit souvent. Françoise Brunel a insisté sur l'héritage institutionnel de la Terreur : le gouvernement révolutionnaire n'a-t-il pas finalement réussi à préserver la continuité de l'État dans une période de crise inédite (p. 34) ? Dans son article historiographique, Haim Burstin, citant les travaux de JeanClément Martin, rappelle que désormais, la Terreur ne peut plus être pensée indépendamment de la contre-révolution, sa sœur ennemie (p. 43). Puis, à la suite de Bronislaw Baczko, il refuse de définir la Terreur comme un projet préconçu, mais comme une réponse à un enlisement, inspirée des expériences révolutionnaires antérieures (p. 45). Selon Alan Forrest, le fait que l'armée soit utilisée pour le maintien de l'ordre, notamment en Vendée, n'est guère une nouveauté dans l'histoire du pays. Elle n'en joue pas moins un rôle particulier dans la répression politique, renforce sa discipline grâce à une justice d'exception assurée par des commissions pour le moins sévères et remplit une mission de plus en plus idéologique. À bien des égards, le Directoire et l'Empire sauront se souvenir de cette terreur militaire. La contribution de David Andress est dissonante : pour lui, la Terreur ne provient pas, comme beaucoup l'ont affirmé depuis Taine, de la nature violente des masses populaires mais de "l'organisation violente de l'État » (p. 78) et de son essence même. La focalisation sur la violence aboutit à une nouvelle naturalisation de la Terreur. Ainsi, lorsque David Andress affirme que c'est toujours au nom de l'État que les violences sont perpétrées, n'oublie-t-il pas que dans bien des cas, c'est aussi au nom de l'État que certains massacres sont évités et que la loi se substitue à la vengeance ? Les articles de Jean-Paul Rothiot, Laurent Brassard et Bernard Bodinier montrent combien les études de cas permettent d'éviter les généralisations. Dans les Vosges comme dans l'Eure ou dans l'Aisne, la Terreur ne s'est jamais appliquée sans compromis ni conciliation. Laurent Brassart, réfutant l'idée d'un centralisme jacobin, ne craint pas d'affirmer que cette période consacre même un apogée des pratiques démocratiques (p. 104): les municipalités axonnaises, jamais vraiment contrôlées par les comités de surveillance ni les sociétés populaires, ont bel et bien été les lieux d'une légère ouverture des institutions municipales aux classes populaires et surtout d'une "Terreur négociée » (p. 109-110).

4 L'ouverture de la deuxième partie («Violences et justice») est délibérément provocatrice. Pour Jean-Clément Martin, la «Terreur» ne fut jamais vraiment mise à l'ordre du jour au sens institutionnel. Bien souvent, elle s'inscrit dans le droit fil de conflits antérieurs et ne relève pas d'un système juridique et politique. Elle correspond plutôt à un mot d'ordre repris par l'historiographie post-thermidorienne pour forger un mythe. Partant, il n'est pas sûr qu'il faille continuer à utiliser ce terme de propagande, mais plutôt celui de "guerre civile", longtemps resté l'impensé de la Révolution, révélant une des causes des violences: le défaut d'État, impuissant à réguler la discorde nationale jusqu'en 1794. Pour Donald Sutherland, qui étudie les 
Bouches-du-Rhône et le Sud-Est, les lynchages populaires de la Terreur résultent de conflits antérieurs peu contrôlés par les autorités. Pourtant, Jacques Guilhaumou et Martine Lapied montrent que dans le même Sud-Est, la Terreur se traduit par un progrès de la politisation et de l'engagement des femmes non dans la violence, mais dans le mouvement sectionnaire, la répression du fédéralisme, la question des subsistances, la bienfaisance sociale, la surveillance morale ou la résistance à la répression politique. Bruno Hervé présente un tableau tout aussi nuancé : en LoireInférieure, la justice révolutionnaire a agi avec discernement et clémence et les autorités locales ont souvent amorti la répression politique, protégeant les communautés contre les ingérences extérieures (p. 16), reconstruisant même le contrat social grâce à une conception pragmatique de la justice d'exception (p. 166). Les conclusions d'Anne Simonin, qui s'attaque au Tribunal révolutionnaire de Paris, correspondent à celles de Robert Allen à propos des tribunaux criminels ordinaires : entre avril 1793 et février-mars 1794, si l'on excepte les mois de vendémiaire et de brumaire an II (de septembre à novembre 1793), les taux d'acquittement de la plus célèbre juridiction d'exception de la Terreur dépassent $50 \%$ (p. 183-184), se maintenant à $19,5 \%$ entre le 23 prairial (10 juin 1794) et le 14 thermidor ( $1^{\text {er }}$ août 1794 ). L'acquittement est un impensé de la justice d'exception pendant la Révolution : depuis deux cents ans, l'historiographie s'est focalisée sur les condamnations. Or Anne Simonin, replaçant son objet dans son contexte particulier, démontre que l'acquittement est une décision judiciaire complexe, qui n'innocente qu'à $41,8 \%$ des prévenus. Les autres sont seulement "libérés ", c'est-à-dire "mis hors de cours " $(49,4 \%)$, déclarés "acquittés-suspects » $(5,2 \%)$ ou même "acquittés-coupables » $(2,4 \%)$. Pourquoi acquitte-t-on? Les crimes à la fois les plus condamnés et les plus acquittés sont les "crimes de paroles ". Pour Anne Simonin, si le crime est le même, c'est la qualification qui change. Alors que les législateurs répriment de plus en plus les propos séditieux, qui atteignent la gravité du crime d'incivisme à partir du printemps 1793 , les jurés, en acquittant les auteurs de ces «mauvaises paroles ", manifestent la résistance de la société civile au peuple et à la langue idéales que les élites révolutionnaires rêvent de forger (p. 202). Sous la Grande Terreur, le Tribunal révolutionnaire, qui transforme des ennemis en amis du peuple participe donc aussi à l'invention d'une dignité citoyenne.

5 Il est simpliste d'expliquer la Terreur par les seules circonstances. Mais on ne peut pour autant minimiser la contrainte économique que l'effort de guerre impose à l'État (Troisième partie, introduction de Gérard Gayot et de Jean-Pierre Jessenne). Période de crise exceptionnelle, la Terreur révèle les inégalités sociales au grand jour. Elle inspire des politiques économiques et sociales parfois très novatrices, qui participent à la construction de l'État Providence. Cet interventionnisme s'accompagne d'une modernisation de l'administration financière et de la comptabilité nationale. Alors qu'on se focalise souvent sur la question du volontarisme politique, les auteurs de l'introduction soulignent enfin l'acceptation du labeur et des privations: les citoyens ordinaires qui contribuent ainsi à l'effort national au nom de la Révolution (p. 220). À Nantes, la crise économique provoque des conflits du travail et une montée des revendications salariales, ferments de la politisation populaire (Samuel Guicheteau). Dans les ateliers d'armurerie parisiens, le consentement à l'effort de guerre se traduit par une augmentation considérable de la productivité du travail, qui permit à l'armée de reprendre l'offensive après les défaites du printemps et de l'été 1793 (Jeff Horn). La nation vit au rythme de la guerre : dans les Pyrénées-Orientales (Thérèse Armengol), 
l'Hérault (Nathalie Alzas) et le Puy-de-Dôme (Bruno Ciotti), les populations sont fortement mobilisées. Dans la lignée des travaux de François Hincker, Claudine Wolikow et Jean-Pierre Gross, Mathieu Soula rappelle que sous la Terreur, les politiques fiscales extraordinaires tentent de corriger les inégalités et de redistribuer les richesses par la progressivité de l'impôt. L'an II, marqué par de grandes lois sociales, voit aussi l'assistance publique se substituer à la charité : la lutte contre la pauvreté devient une mission de l'État (Danièle Pingué et Armelle Ponsot). Enfin, malgré la grande loi du 17 juillet 1793 qui abolit la féodalité, Serge Aberdam rappelle que la Terreur ne se traduit par aucune politique agraire générale dans le long terme. Si le débat sur le partage des terres et les transferts de propriété continue à faire rage, le gouvernement révolutionnaire mène avant tout une politique d'urgence, dans le cadre d'une économie de guerre (p. 300), que ce colloque remet donc au premier plan.

6 La quatrième partie, consacrée à la vie culturelle et religieuse, est la plus hétéroclite. Contre des clichés encore très répandus, les communications interdisent d'associer la Terreur à une période de déclin culturel et scientifique dans laquelle l'ensemble de la pensée et de la création aurait été entièrement contrôlée par l'État. Diffusion du théâtre engagé, apogée de la littérature militante, essor des fêtes civiques et de la peinture d'actualité: une "frénésie créatrice» (Philippe Bourdin, introduction) favorise l'émergence d'une nouvelle génération d'artistes, d'hommes de lettres et de savants connus ou non, aux identités multiples, groupe hétéroclite qui se dessine derrière les élites, jusqu'ici davantage étudiées. L'étude de cas proposée par Éric Saunier, consacrée au Précis de la Révolution relativement à Louis 16. Respec du aux Rois et aux Puissances de François-Toussaint Bonvoisin, négociant havrais, montre ce que l'étude des petites plumes de la Révolution, dont les stratégies d'écriture s'intègrent dans des pratiques sociales et professionnelles multiples, peut apporter à l'histoire des différents modes d'engagement: le texte n'est ici pas analysé comme un simple témoignage, ni comme un écrit du for privé, mais au contraire comme une action intimement liée aux événements eux-mêmes : alors que souvent, les historiens se sont penchés sur le monde du commerce dans le cadre d'études économiques, E. Saunier montre que le Précis est rédigé en réaction à la politique religieuse du gouvernement révolutionnaire. Dans le même esprit, l'article de Jean-Luc Chappey convaincra de l'utilité des approches sociales dans l'histoire des sciences. Alors que les "savants" sont souvent définis à travers des identités fixes, réduits à leurs activités intellectuelles et scientifiques, la mise en valeur de leurs différents engagements fait surgir de nouvelles figures aux combinaisons multiples (le savant artiste, le savant écrivain, le savant politique) et remet en cause des visions trop générales. Loin d'entraîner un déclin des savoirs scientifiques ou un contrôle politique des savants, la Terreur accélère la spécialisation des savoirs et renforce l'autorité de l'élite scientifique, dont les membres sont tenants d'une "science sévère" et acteurs de nouvelles institutions ouvertes à une minorité de spécialistes. Selon J-L. Chappey, ce cloisonnement des savoirs, conçu en 1793-1794 comme une arme temporaire pour sauver la Révolution, sera repris par Bonaparte à partir de 1799, dans le but inverse de terminer celle-ci... Serge Bianchi rappelle quant à lui que l'iconoclasme révolutionnaire, destruction ciblée, s'accompagne de la constitution et de la sauvegarde d'un nouveau patrimoine national. Enfin, une série d'articles aborde les problèmes religieux, cruciaux tant la Terreur est souvent associée à une répression antichrétienne. Daniel Schönpflung, montre qu'à Strasbourg, la nouvelle religion civique est une initiative des élites politiques locales même si de son côté, Jacques Bernet rappelle combien la 
déchristianisation de l'an II est localement imputée aux factions politiques ou au pouvoir central: l'exemple des seize carmélites de Compiègne, condamnées pour collusion avec Catherine Théot et dom Gerle, montre que la politique antichrétienne dissimule des luttes plus politiciennes. La figure de Sylvain Maréchal, étudiée par Erica Joy Man-nucci, révèle le rôle de la Terreur dans la laïcisation de la vie politique. Enfin, Stéphane Baciocchi et Philippe Boutry proposent de revenir sur des sources au maniement très délicat, car forgées par la mémoire contre-révolutionnaire : celles des listes des « victimes » ecclésiastiques de la Terreur et de leurs martyrologes. Comme le soulignent les deux auteurs, cette prosopographie implique une profonde réflexion sur les catégories généralement employées, forgées par les milieux chrétiens («victimes », «martyrs », " persécutions »), si l'on veut dénombrer les individus concernés. Malgré leur opacité, les martyrologues permettent de montrer que $72 \%$ des prêtres condamnés au Tribunal révolutionnaire de Paris sont des constitutionnels : les réfractaires, dont le cas, plus simple à juger, pose moins de difficulté, sont en général jugés localement.

7 En conclusion, Claude Mazauric appelle à relativiser la spécificité française de la Terreur comme violence d'État et souligne son cadre légal. La Terreur est donc bien plus complexe que la tyrannie ou le régime pré-totalitaire qu'on continue parfois de caricaturer. Comme période révolutionnaire, la Terreur est politique, mais elle dépasse de loin la violence d'État et la politique répressive. C'est ce que démontre ce colloque, certes presque entièrement pensé à décharge. Vingt ans après les durs combats du Bicentenaire, le processus de normalisation historiographique de la Terreur commence à peine. 